\title{
Analytic Approximations for Thomas-Fermi Equation
}

\author{
A. El-NahHas \\ Department of Mathematics, Helwan Faculty of Science \\ Helwan, Egypt \\ (Received February 12, 2008; revised version March 28, 2008; \\ in final form April 29, 2008)
}

In this paper, we give an analytic approximation to the solution of the Thomas-Fermi equation using the homotopy analysis method and with the use of a polynomial exponential basis.

PACS numbers: 02.30.Hq, 02.30.Mv, 02.60.Lj, 21.10.Ft, 31.15.-p

\section{Introduction}

The Thomas-Fermi equation presented in the Thomas-Fermi atom model $[1,2]$ has the form

$$
\ddot{u}(t)=\sqrt{\frac{u^{3}(t)}{t}}
$$

and with the boundary conditions

$$
u(0)=1, \quad u(\infty)=0 .
$$

This equation describes the spherically symmetric charge distribution for a multielectron atom. It has a singularity at $t=0$ and was dealt by a group of methods, see e.g., [3-16], but most of them are not purely analytic. The homotopy analysis method is a powerful tool introduced by Liao $[17,18]$ to give analytic approximations for nonlinear problems. Unlike other methods such as perturbation $[19,20]$ which depends upon the existence of small or large parameters in the studied equation and the Adomian decomposition method [21] which gives local convergent regions for the solutions, the homotopy analysis method does not depend upon small parameters and proposes a direct way to control the convergence of approximation series. This method was applied by Liao [22], for the ThomasFermi equation, to give a purely analytic solution for this equation using a fractional basis. Also, Liao proved a convergence theorem concerning the solutions of the Thomas-Fermi equation in this article. Here we apply the homotopy analysis method, for the same equation, to investigate its analytic solution, but with the use of a polynomial exponential basis. 
A transformation of the form

$$
x=1+\lambda t,
$$

where $\lambda$ is a parameter to be determined later, transforms Eq. (1) to the equation

$$
\lambda^{3}(x-1)\left[u^{\prime \prime}(x)\right]^{2}-u^{3}(x)=0, \quad ' \equiv \frac{\mathrm{d}}{\mathrm{d} x}
$$

with the new boundary conditions

$$
u(1)=1, \quad u(\infty)=0 .
$$

Equation (4) is one which has a strong nonlinearity. We apply the homotopy analysis method to this equation and then deduce the corresponding results for Eq. (1). The parameter $\lambda$, in Eq. (4), is chosen such that we have best approximations.

\section{Application of the homotopy analysis method}

In the application of the homotopy analysis method to Eq. (4) and considering the conditions (5), we take the polynomial exponential basis

$$
\left\{x^{m} \mathrm{e}^{-n x}, \quad m \geq 0, \quad n \geq 1\right\} .
$$

Since Eq. (4) contains the variable $x$, this choice of the basis is reasonable then if we choose the exponential basis only.

We express the desired solution by the rule of expression

$$
u(x)=\sum_{n=1}^{\infty} \sum_{m=0}^{\infty} A_{n, m} x^{m} \mathrm{e}^{-n x} \quad\left(A_{n, m} \text { are coefficients }\right) .
$$

According to (4), (5), (6) and (7), we choose, respectively, the initial approximation and the auxiliary linear operator as follows:

$$
\begin{aligned}
& u_{0}(x)=\mathrm{ee}^{-x}, \\
& L=\frac{\partial^{2}}{\partial x^{2}}+\frac{\partial}{\partial x} .
\end{aligned}
$$

We construct the so-called zero-order deformation equation

$$
(1-q) L\left[\phi(x, q)-u_{0}(x)\right]=h H(x) q N[\phi(x, q)]
$$

subject to the boundary conditions

$$
\phi(1, q)=1, \quad \phi(\infty, q)=0
$$

where $h$ is an auxiliary parameter, $H(x)$ is an auxiliary function and $q$ is an embedding parameter varied from 0 to 1 . As $q$ varies from 0 to 1 the solution of Eq. (10) varies from the initial approximation to the exact solution of Eq. (4).

Thus we have

$$
\phi(x, 0)=u_{0}(x), \quad \phi(x, 1)=u(x) .
$$

In Eq. (10), $N$ is a nonlinear operator defined as

$$
N[\phi(x, q)]=\lambda^{3}(x-1)\left[\frac{\partial^{2} \phi(x, q)}{\partial x^{2}}\right]^{2}-\phi^{3}(x, q) .
$$


By means of the Taylor power series, at $q=0$, we have

$$
\phi(x, q)=\phi(x, 0)+\sum_{m=1}^{\infty} u_{m}(x) q^{m}
$$

where

$$
u_{m}(x)=\left.\frac{1}{m !} \frac{\partial^{m} \phi(x, q)}{\partial q^{m}}\right|_{q=0} .
$$

If the parameter $h$ is properly chosen such that the series (14) is convergent at $q=1$, then from (12)

$$
u(x)=u_{0}(x)+\sum_{m=1}^{\infty} u_{m}(x) .
$$

Now differentiating the zero-order deformation Eq. (10) $m$ times with respect to $q$ and setting $q=0$ and finally dividing it by $m$ !, we obtain the $m$-th order deformation equation

$$
L\left[u_{m}(x)-\chi_{m} u_{m-1}(x)\right]=h H(x) R_{m}(x), \quad m \geq 1,
$$

where

$$
\begin{aligned}
& R_{m}(x)=\sum_{n=0}^{m-1}\left[\lambda^{3}(x-1) u_{n}^{\prime \prime}(x) u_{m-1-n}^{\prime \prime}(x)-u_{m-1-n}(x) \sum_{i=1}^{n} u_{i}(x) u_{n-i}(x)\right], \\
& \chi_{m}= \begin{cases}0, & m=1, \\
1, & m>1 .\end{cases}
\end{aligned}
$$

The solutions of the Eq. (17) are called the $m$-th deformations, $m \geq 1$, and these deformations may satisfy the boundary conditions

$$
u_{m}(1)=0, \quad u_{m}(\infty)=0, \quad m \geq 1 .
$$

The auxiliary function $H(x)$ may be chosen such that there is no contradiction with the rule of expression (7), that is no strange terms appear in the subsequent deformations from that of this rule. Also this choice may be consistent with the rule of coefficient ergodicity, that is coefficients in (7) can be modified to ensure the completeness of the set of base functions.

The solutions of Eq. (17) can be obtained by a symbolic software such as Mathematica, and it results that its solutions can be expressed as

$$
u_{m}(x)=\sum_{n=1}^{2 m+1} \sum_{k=0}^{2 m+1} a_{m, n}^{k} x^{k} \mathrm{e}^{-n x} \quad\left(a_{m, n}^{k} \text { are coefficients }\right)
$$

For a reasonable choice of $H(x), \lambda$ and $h$ the $M$-th order approximation for the solution of Eqs. (4),(5) is then

$$
U(x, M)=u_{0}(x)+\sum_{m=1}^{M} \sum_{n=1}^{2 m+1} \sum_{k=0}^{2 m+1} a_{m, n}^{k} x^{k} \mathrm{e}^{-n x}
$$


and consequently the $M$-th order approximation for the solution of Eqs. (1), (2) takes the form

$$
U(t, M)=u_{0}(1+\lambda t)+\sum_{m=1}^{M} \sum_{n=1}^{2 m+1} \sum_{k=0}^{2 m+1} a_{m, n}^{k}(1+\lambda t)^{k} \mathrm{e}^{-n(1+\lambda t)} .
$$

The totally analytic solution of Eqs. (1), (2) is

$$
u(t)=\lim _{M \rightarrow \infty} U(t, M)
$$

The best values of both the parameters $\lambda$ and $h$ which control the convergence of the approximations of kind (23) can be studied by investigating the convergence of the series of $u^{\prime}(0)$ and plotting the so-called $h$-curve which takes a horizontal line through the position of convergence.

\section{Results of the application}

It is found that the auxiliary function $H(x)$ can be chosen to take the value, $H(x)=1$, to get best consistent analytic approximations. Computing these approximations up to the 30th order and plotting the curve of $u^{\prime}(0)$ versus $\lambda$ for different values of the parameter $h$, we gain the fact that $\lambda$ can be chosen to take the value $\lambda=0.5$. Figure 1 depicts the curve of the parameter $\lambda$ versus $u^{\prime}(0)$ at $h=-0.2$ (thin line), $h=-0.5$ (solid line) and $h=-0.8$ (dashed line), respectively. The curve of $u^{\prime}(0)$ versus $h$ at $\lambda=0.5$ is plotted in Fig. 2, and from this curve we choose $h=-0.5$. The curve representing the solution of the Thomas-Fermi Eqs. (1), (2) is traced in Fig. 3, for $h=-0.5$ and $\lambda=0.5$. Figure 2 indicates that the series (23) diverges as $M \rightarrow \infty$ when $h \rightarrow-1$, while we have a good convergence at $h=-0.5$. Also at $h=-0.5$, we have the value $u^{\prime}(0)=-1.55167$.

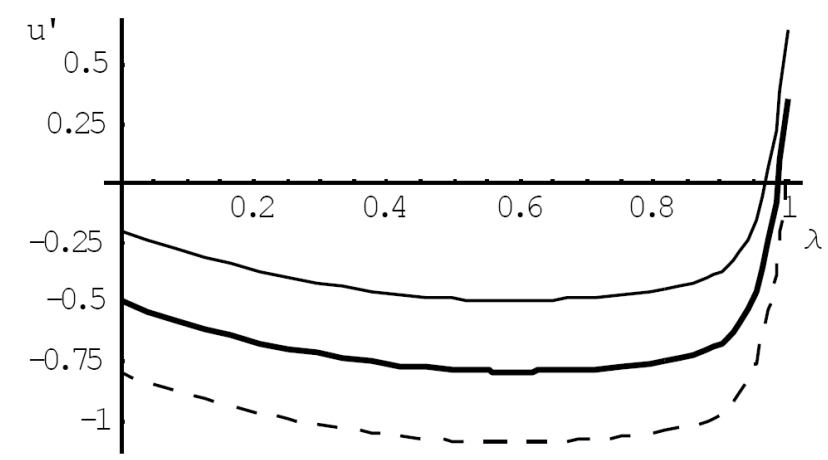

Fig. 1. The curve of $u^{\prime}(0)$ versus $\lambda$ at $h=-0.2,-0.5,-0.8$.

As pointed by Liao [22], the energy of a neutral atom in the Thomas-Fermi model is determined by the equation

$$
E=\frac{6}{7}\left(\frac{4 \pi}{3}\right)^{2 / 3} Z^{7 / 3} u^{\prime}(0)
$$




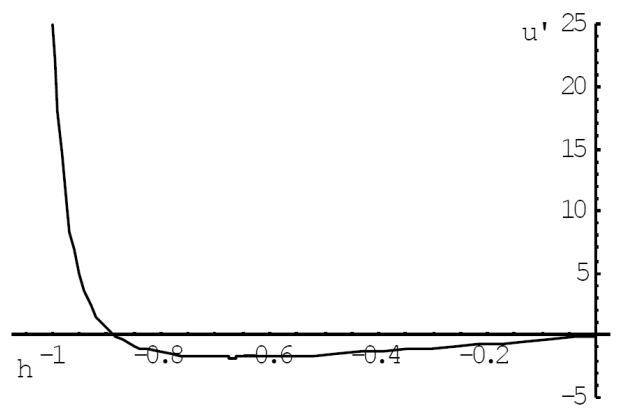

Fig. 2. The $h$ curve of $u^{\prime}(0)$ at $\lambda=0.5$.

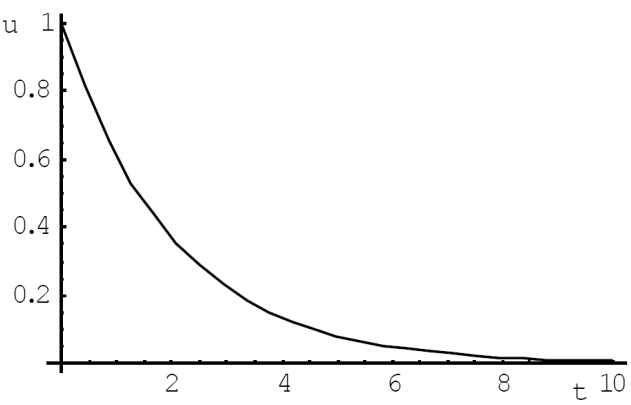

Fig. 3. The solution of Thomas-Fermi equation.

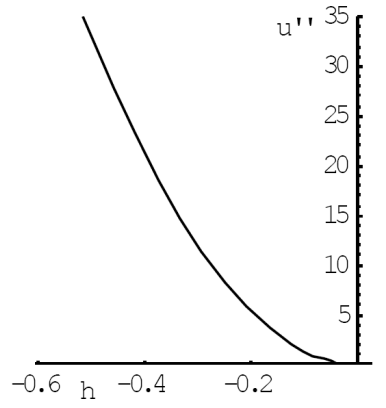

Fig. 4. The $h$ curve of $u^{\prime \prime}(0)$ at $\lambda=0.5$.

where $Z$ is the charge. The value $u^{\prime}(0)$ in Eq. (25) which represents the initial slope of the Thomas-Fermi equation, was computed by Kobayashi [23] to be $u^{\prime}(0)=$ -1.58807 , which can be approached via increasing the order of approximation. Figure 4 investigates the $h$-curve of $u^{\prime \prime}(0)$, and we have the value $u^{\prime \prime}(0)=33.25$ at $h=-0.5$. This value increases rapidly as the order of approximation increases and is consisting with the fact that $u^{\prime \prime}(0) \rightarrow \infty$ as $t \rightarrow 0$, which follows from Eq. (1). Thus we cannot use $u^{\prime \prime}(0)$ to control the convergence of the approximation series. 


\section{Conclusion}

This paper used the homotopy analysis method to obtain analytic approximations for the Thomas-Fermi equation via a polynomial exponential basis and proved the flexibility and the great ability of this method to deal nonlinear problems for any set of base functions and also to control the convergence of resulting approximation series with a high degree of efficiency.

\section{Acknowledgments}

The author thanks the anonymous reviewer for his valuable suggestions.

\section{References}

[1] E. Fermi, Rend. Accad. Naz. del Lincei, Cl. Sci. Fis., Mat. E. Nat. 6, 602 (1927).

[2] L.H. Thomas, Proc. Cambridge Philos. Soc. 23, 542 (1927).

[3] M. Allan, Comp. Phys. Commun., 67, 389 (1992).

[4] B.L. Burrows, P.W. Core, Quart. Appl. Math. 42, 73 (1984).

[5] V. Bush, S.H. Caldwell, Phys. Rev. 38, 1898 (1931).

[6] C.Y. Chan, S.W. Du, Quart. Appl. Math. 44, 303 (1986).

[7] C.Y. Chan, Y.C. Hon, Quart. Appl. Math. 45, 591 (1987).

[8] F. Civan, C.M. Sliepcevich, J. Comput. Phys. 56, 343 (1984).

[9] Y.C. Hon, SEA Bull. Math. 20, 55 (1996).

[10] B.J. Laurenzi, J. Math. Phys. 31, 2535 (1990).

[11] C.D. Luning, W.L. Perry, Quart. Appl. Math. 35, 257 (1977).

[12] K.A. Milton, C.M. Bender, S.S. Pinsky, J. Math. Phys. 30, 1447 (1989).

[13] G.J. Pert, J. Phys. B 32, 5067 (1999).

[14] S.N. Venkatarangan, K. Rajalashmi, Comput. Math. Appl. 29, 75 (1995).

[15] A.M. Wazwaz, Math. Comput. 105, 11 (1999).

[16] M.S. Wu, Phys. Rev. A 26, 57 (1982).

[17] S.J. Liao, Ph.D. Thesis, Shanghai Jiao Tong University, 1992.

[18] S.J. Liao, Beyond Perturbation: Introduction to the Homotopy Analysis Method, Chapman\&Hall/CRC Press, Boca Raton 2003.

[19] J.D. Cole, Perturbation Methods in Applied Mathematics, Blaisdell Publ. Co., Waltham (MA) 1968.

[20] A.H. Nayfeh, Perturbation Methods, Wiley, New York 2000.

[21] G. Adomian, Solving Frontier Problems of Physics: The Decomposition Method, Kluwer Academic Publ., Boston 1994.

[22] S.J. Liao, Appl. Math. Comput. 144, 495 (2003).

[23] S. Kobayashi, T. Matsukuma, S. Nagai , K. Umeda, J. Phys. Soc. Jpn. 10, 759 (1955). 